\title{
Decreased Stathmin-1 Expression Inhibits Trophoblast Proliferation and Invasion and Is Associated with Recurrent Miscarriage
}

\author{
Fu-Ju Tian, ${ }^{* \dagger}$ Chuan-Mei Qin,${ }^{* \dagger}$ Xiao-Cui Li, ${ }^{*}$ Fan Wu, ${ }^{* \dagger}$ Xiao-Rui Liu, ${ }^{* \dagger}$ Wang-Ming $\mathrm{Xu},{ }^{\ddagger}$ and $\mathrm{Yi} \mathrm{Lin}{ }^{* \dagger}$
}

From the International Peace Maternity and Child Health Hospital* and the Institute of Embryo-Fetal Original Adult Disease, ${ }^{\dagger}$ Shanghai Jiao Tong University School of Medicine, Shanghai, China; and the Reproductive Medicine Center ${ }^{\ddagger}$ Renmin Hospital of Wuhan University, Wuhan, China

\author{
Accepted for publication \\ June 18, 2015. \\ Address correspondence to $\mathrm{Yi}$ \\ Lin, M.D., International Peace \\ Maternity and Child Health \\ Hospital, Shanghai Jiao Tong \\ University School of Medicine, \\ No. 910, HengShan Rd, \\ Shanghai 200030, China; or \\ Wang-Ming Xu, M.M.Sc., \\ Reproductive Medicine Center, \\ Renmin Hospital of Wuhan \\ University, No. 99, Zhang \\ Zhidong Rd, Wuhan 430060, \\ China. E-mail: yilinonline@ \\ 126.com or wangmingxu118@ \\ 163.com.
}

\begin{abstract}
Fetal trophoblasts invade endometrium and establish a complex interaction with the maternal microenvironment during early pregnancy. However, the molecular mechanisms regulating trophoblast migration and invasion at the maternal-fetal interface remain poorly understood. Immunohistochemistry and immunoblotting have shown that stathmin-1 (STMN1) was down-regulated significantly in placental villi tissue and trophoblasts from patients with recurrent miscarriage. In vitro, overexpression of STMN1 promoted human trophoblast proliferation, migration, and invasion, whereas knockdown of STMN1 inhibited these processes. In addition, knockdown of STMN1 down-regulated $\mathrm{N}$-cadherin and upregulated $\mathrm{E}$-cadherin in trophoblasts, whereas $\mathrm{E}$-cadherin was up-regulated and $\mathrm{N}$-cadherin was downregulated in recurrent miscarriage villi tissue. Knockdown of STMN1 attenuated cytoplasmic-nuclear translocation of $\beta$-catenin and in turn down-regulated trophoblast matrix metalloproteases. Furthermore, tumor necrosis factor- $\alpha$ (TNF- $\alpha$ ) down-regulated STMN1 expression, and serum TNF- $\alpha$ expression correlated inversely with trophoblast STMN1 levels. Interestingly, M1 macrophage-derived TNF- $\alpha$ reduced trophoblast migration and invasion, and an anti-TNF- $\alpha$ antibody reversed this effect. Collectively, this study indicated that STMN1 may play a key role in regulating trophoblast invasion, and that impaired STMN1 expression may lead to abnormal trophoblast invasion and result in recurrent miscarriage. (Am J Pathol 2015, 185: 2709-2721; http://dx.doi.org/10.1016/j.ajpath.2015.06.010)
\end{abstract}

The delicate chorus between fetal trophoblasts and maternal cells is one of the most fascinating biological processes in normal pregnancy. ${ }^{1}$ Successful embryo implantation depends on embryo hatching, trophoblast development, and proper maternal-fetal cross-talk and immune regulation. The invasive potential of trophoblasts is essential for embryo implantation. ${ }^{2}$ Insufficient proliferation and invasion of cytotrophoblasts is associated with preeclampsia (PE), fetal growth restriction, and recurrent miscarriage (RM). ${ }^{3,4} \mathrm{RM}$ affects approximately $1 \%$ to $3 \%$ of females during their reproductive years, and usually is defined as three or more consecutive spontaneous abortions before 20 weeks of gestation. ${ }^{5} \mathrm{RM}$ is associated with a high level of psychological distress; therefore, comprehensive molecular studies are required to understand the causes of RM and to develop potential molecular targets.

During implantation and pregnancy, the invading fetal trophoblast population is in close contact with maternal immune cells. ${ }^{6}$ Immune cells, including natural killer cells, $\mathrm{T}$ lymphocytes, and macrophages, produce cytokines, chemokines, growth factors, and hormones to establish a unique maternal-fetal immune microenvironment that contributes to fetal survival and development in the maternal uterus. $1,7,8$ Decidual macrophages represent $20 \%$ to $25 \%$ of the total leukocyte population in the first-trimester decidual tissue

Supported by National Basic Research Program of China grants 2013CB967404 (Y.L.) and 2011CB944400 (W.-M.X.); National Natural Science Foundation of China grants 81401218 (F.-J.T.), 81125004 (Y.L.), and 31171439 (Y.L.); Fund for Outstanding Academic Leaders in Shanghai, China, grants 12XD1406600 (Y.L.) and 2013-049 (Y.L.); the Shanghai Natural Science Fund Project grant 14ZR1443800 (F.-J.T.); and Shanghai Jiao Tong University Medicine-Engineering Fund grant YG2013ZD04 (Y.L.).

F.-J.T. and C.-M.Q. contributed equally to this work.

Disclosures: None declared. 
and remain present throughout pregnancy. ${ }^{9}$ In specific environments, macrophages can be stimulated and polarized into the M1 or M2 subtype. ${ }^{10}$ M1 macrophages are potent effector cells that produce copious amounts of proinflammatory cytokines and mediate inflammatory responses. In contrast, M2 cells regulate inflammatory responses and promote tissue remodeling and repair. ${ }^{11}$ It has been reported that in early pregnancy decidual macrophages can be polarized and differentiated into the M2 subtype, which secrete immunosuppressive cytokines such as IL-10, which play a role in maintaining immune tolerance. ${ }^{12}$ However, recent reports have suggested that decidual macrophages polarize into the M1 subtype and release tumor necrosis factor- $\alpha$ (TNF- $\alpha)$, IL- 6 , and IL-1 $\beta$ to promote RM. ${ }^{13}$ However, it remains unknown whether abnormal interactions between trophoblasts and macrophages dysregulate trophoblast function and promote RM.

Stathmin-1 (STMN1), also known as oncoprotein 18, is encoded by human STMN1, located on chromosome $1 .{ }^{14}$ STMN1 is a major cytosolic phosphoprotein that regulates microtubule dynamics by preventing tubulin polymerization and promoting microtubule destabilization via both sequestration of $\alpha / \beta$-tubulin heterodimers and enhancement of microtubule catastrophe. ${ }^{15}$ STMN1 is involved in tumor cell proliferation, invasion, differentiation, and motility. ${ }^{16}$ STMN1 dysfunction can lead to constant microtubule assembly and uncontrolled cell-cycle progression, which can result in abnormal cell growth and tumor formation. ${ }^{17,18}$ Recent reports have shown that STMN1 is expressed highly in rodent placental tissue, villous cytotrophoblasts, and extravillous trophoblasts, but not in syncytiotrophoblasts in first-trimester human placenta. ${ }^{19,20}$

Here, we report that the down-regulation of STMN1 decreases trophoblast proliferation and invasion by regulating the E-cadherin/ $\beta$-catenin pathway, and that STMN1 expression is regulated negatively by TNF- $\alpha$ derived from M1 macrophages. In patients with RM, STMN1 expression was downregulated in chorionic villi and correlated negatively with the serum TNF- $\alpha$ level. Our results suggest that STMN1 is a trophoblast proliferation - and invasion-associated microtubule regulatory protein that participates in the pathogenesis of RM.

\section{Materials and Methods}

\section{Patient Characteristics}

Fifteen patients with RM, aged 22 to 37 years (mean age, $30.1 \pm 6.0$ years), who were treated at the Department of Obstetrics and Gynecology in the International Peace Maternity and Child Health Hospital of China Welfare Institute at Shanghai Jiao Tong University School of Medicine between July 2013 and July 2014 were included in this study. Patients with the following features were excluded: i) absence of uterine abnormality or cervical incompetence on pelvic examination and ultrasound; ii) abnormal karyotype analysis of the parents or abortus; iii) comprehensive hormonal status assessment to rule out luteal phase defects, hyperprolactinemia, and hyperandrogenemia; and iv) no symptoms of endocrine or metabolic diseases (diabetes, hyperthyroidism, and hypothyroidism). Specimens of villi tissue were collected and stored in liquid nitrogen.

In addition, 16 women aged 22 to 35 years (mean age, $27.9 \pm 3.9$ years) with normal early pregnancies were recruited as healthy controls (HCs). All of these women had at least one successful pregnancy without spontaneous abortion, preterm labor, or PE in any pregnancy. These patients underwent artificial abortion to terminate their unwanted pregnancies at 8 to 12 weeks' gestation and samples of villi tissue were collected and stored in liquid nitrogen.

PE was defined as new onset of both hypertension (systolic/diastolic blood pressure $\geq 140 / 90 \mathrm{mmHg}$ measured on two occasions at least 4 hours apart) and proteinuria ( $\geq 300 \mathrm{mg}$ per 24 hours) after 20 weeks' gestation. No patients with PE involved in this study had other complications such as obesity, chronic hypertension, gestational diabetes mellitus, or eclampsia. All patients with PE were aged 25 to 34, developed hypertension and proteinuria after a gestational age of 32 weeks, and delivered at 36 to 40 weeks, which matched with normal controls. Informed consent for placenta donation was obtained from all women.

The protocol of this study was approved by the Medical Ethics Committee of the International Peace Maternity and Child Health Hospital of China Welfare Institute (Shanghai, China). Written informed consent was obtained from all participants before enrollment.

\section{Cell Culture}

Primary trophoblasts were isolated by trypsin-DNase I digestion and discontinuous Percoll gradient centrifugation from pooled villi obtained from five to eight patients, as previously described. ${ }^{21}$ Trophoblasts were seeded in cell culture plates for further purification based on differential adherent velocity to eliminate adherent fibroblast cells and unattached leukocytes. This method resulted in approximately $95 \%$ pure cultures of trophoblasts, as assessed by flow cytometry for cytokeratin 7 positivity, human leukocyte antigen $-\mathrm{G}$ positivity, and vimentin negativity (data not shown). Purified trophoblasts were seeded in 12-well plates at $1 \times 10^{6}$ cells $/ \mathrm{mL}$ for further experiments.

The HTR-8/SVneo cell line, ${ }^{22}$ derived from human invasive extravillous trophoblasts, was a generous gift from Dr. Peeyush K. Lala (University of Western Ontario, London, ON, Canada). Cells were cultured in Dulbecco's modified Eagle's medium (DMEM) (high-glucose medium)/F12 plus $10 \%$ FBS (Gibco, Grand Island, NY) with antibiotics.

\section{Confocal Imaging}

HTR-8 cells were cultured on poly-L-lysine-coated coverslips in 24-well plates, transfected with control siRNA (siCtrl) or siRNA specific to STMN1 (siSTMN1), cultured for 48 hours, 
washed three times with phosphate-buffered saline (PBS), fixed in $4 \%$ paraformaldehyde-PBS for 10 minutes, and stained with primary antibodies [1:200 dilution for all antibodies, except anti-STMN1 (1:500)] using standard immunofluorescence protocols. Images were captured and assessed using a Leica (Wetzlar, Germany) SP8 confocal microscope.

\section{Overexpression of STMN1}

To generate the STMN1-overexpressing construct, the coding region sequence (CDS) of human STMN1 was cloned into pLVX-IRES-ZsGreen vector (Clontech Laboratories, Mountain View, CA) using the following primers: CDS 5'-ATGAATTCCTTGTCTTCTATTCACCATGGCTTC-3' (forward) and $5^{\prime}$-CCGGGATCCTTAAGTACCTAGTGATGGTGAG$3^{\prime}$ (reverse). All constructs were verified by sequencing (Life Technologies, Grand Island, NY). PLVX-IRESZsGreen-STMN1 and control vector were purified using the Endofree Plasmid Kit (Qiagen, Duesseldorf, Germany), and transfected into the cells using Lipofactamine 3000 (Life Technologies).

\section{Knockdown of STMN1}

Knockdown of STMN1 was performed using specific siSTMN1 oligonucleotides. Unless otherwise indicated, all oligos were purchased from GenePharma, Inc. (Shanghai, China), and transfected into the cells at a final concentration of $100 \mathrm{nmol} / \mathrm{L}$ using Oligofectamine reagent (Invitrogen, Grand Island, NY).

\section{Cell Proliferation Assay}

For cell proliferation studies, HTR-8 cells were plated at $2 \times 10^{3}$ cells/well in 96-well plates. Cell viability was determined at 24, 48, 72, or 96 hours using the MTT assay (Sigma, St. Louis, MO). Absorbance was measured at $490 \mathrm{~nm}$ using a Spectra Max 190 microplate reader (Bio-Rad, Hercules, CA).

\section{Wound Healing Assay}

HTR-8 cells were transfected with siCtrl, siRNA, empty vector, or the STMN1-overexpressing plasmid. After 48 hours, the cells reached approximately $80 \%$ confluence. Then, a sterile 1-mL pipette tip was used to scrape a cross in the center of the monolayer, and the cells were rinsed three times with ice-cold PBS and cultured in serum-free medium. The cells were allowed to migrate for 24 hours, and the gaps were observed and photographed. Light and fluorescent microscopic images were captured using a Leica DMI 3000B microscope. The sizes of the gaps were measured from the photomicrographs.

\section{Quantitative Real-Time PCR}

Total RNA was extracted from cultured cells or primary cells using TRIzol reagent (Life Technologies) according to the manufacturer's instructions, and was used to generate cDNA with the PrimeScript II first Strand cDNA Synthesis Kit (Takara, Dalian, China) using random or oligo-dT primers. Quantitative real-time PCR was performed using a SYBR Green kit (Takara). The primers were as follows: STMN1 $5^{\prime}$-AGAACCGAGAGGCACAAATG-3' (forward) and $5^{\prime}$ AGTCAGCAGGGTCTTTGGATT-3' (reverse); GAPDH 5'CACTGGGCTACACTGAGCAC-3' (forward) and $5^{\prime}$-AGTGGTCGTTGAGGGCAAT-3' (reverse). For in vitro experiments, the relative expression was calculated using the $2^{-\Delta \Delta \mathrm{Ct}}$ method and normalized to the internal control gene GAPDH (human). For clinical data, relative expression was calculated using the $2^{-\Delta \mathrm{Ct}}$ method and normalized to the internal control gene GAPDH (human).

\section{Nuclear Protein Extraction and Western Blot Analysis}

Nuclear protein extracts were prepared from trophoblasts using the Nuclear Extraction Kit (Pierce, Rockford, IL) according to the manufacturer's protocol. Antibodies against STMN1 (dilution 1:1000; Abcam, Cambridge, UK), $\beta$-catenin, E-cadherin, and N-cadherin (dilution 1:1000, Epithelial-Mesenchymal Transition Antibody Sampler Kit; Cell Signaling Technology, Beverly MA) were used to perform Western blot analysis using standard techniques. Antibodies against GAPDH or Lamin B (dilution 1:2000; Santa Cruz, Dallas, TX) were used as loading controls.

\section{Immunohistochemistry}

Immunohistochemical staining of human villi or placenta tissue was performed as previously described ${ }^{23}$ using rabbit antiSTMN1 (dilution 1:500; Abcam), anti-E-cadherin (dilution 1:250; Cell Signaling Technology, Inc.), and anti-N-cadherin (dilution 1:1000; Cell Signaling Technology, Inc.).

\section{Human MMP Array}

Briefly, HTR-8 cells were transfected with siCtrl or siSTMN1 oligos, cultured for 48 hours, and then $100-\mu \mathrm{L}$ aliquots of the cell supernatants were harvested and used to assess matrix metalloprotease (MMP) and tissue inhibitor of matrix metalloprotease (TIMP) expression using the Quantibody Human MMP Array 1 (Raybiotech, Inc., Norcross, GA).

\section{Macrophage Purification and Generation}

Umbilical cord blood mononuclear cells were isolated by standard Ficoll-Paque density-gradient (Amersham Biosciences, Pittsburgh, PA), and CD $14^{+}$monocytes were isolated by magnetic-activated cell sorting using a human $\mathrm{CD}^{+}{ }^{+}$microBeads isolation kit (Miltenyi Biotec, Cologne, Germany). The purity of isolated $\mathrm{CD} 14^{+}$monocytes was $>95 \%$, as assessed by flow cytometry. CD14 ${ }^{+}$monocytes were cultured in RPMI 1640 medium (Gibco) supplemented with $10 \%$ fetal bovine serum (Gibco) and $20 \mathrm{ng} / \mathrm{mL}$ 
granulocyte-macrophage colony-stimulating factor. To generate M1 macrophages, day 4 macrophage cultures were activated with $200 \mathrm{ng} / \mathrm{mL}$ lipopolysaccharide (LPS) (Sigma) plus $10 \mathrm{ng} / \mathrm{mL}$ interferon- $\gamma($ IFN- $\gamma$ ) for 48 hours. To generate M2a, M2b, and M2c macrophages, day 5 macrophage cultures were exposed to $50 \mathrm{ng} / \mathrm{mL}$ IL-4 (M2a), IL-1 (M2b), or IL-10 (M2c) for 48 hours, respectively. All cytokines were obtained from PeproTech, Inc.

\section{Invasion Assay}

The invasive ability of trophoblasts across extracellular matrix was evaluated using the Transwell Matrigel invasion assay, as previously described. ${ }^{24}$ Cell culture inserts $(8-\mu \mathrm{m}$ pore size, 6.5-mm diameter; Corning Inc., Corning, NY) were coated with $25 \mu \mathrm{L}$ of Matrigel (Corning) and placed in 24 -well plates. Two sets of invasion assays were performed as follows.

HTR-8 cells were transfected with siCtrl, siSTMN1 oligonucleotides, control vector, or the STMN1-overexpressing vector, cultured for 48 hours, and then $1 \times 10^{5}$ cells in $200 \mu \mathrm{L}$ of DMEM/F12 were placed into the upper chamber of each insert. The lower chambers were filled with $800 \mu \mathrm{L}$ of DMEM/F12 containing 10\% fetal bovine serum, and the plates were incubated at $37^{\circ} \mathrm{C}$ for 48 hours.

A macrophage and trophoblast co-culture invasion model was established to determine the effects of macrophagederived cytokines on the invasive ability of trophoblasts. Briefly, $2.5 \times 10^{5}$ differentiated macrophages (M0, M1, M2a, M2b, or M2c; in $800 \mu \mathrm{L}$ of DMEM containing $15 \%$ fetal bovine serum) were seeded into the lower chambers and cultured for 24 hours at $37^{\circ} \mathrm{C}$. Then, freshly isolated trophoblasts $\left(1 \times 10^{5}\right.$ in $200 \mu \mathrm{L}$ of DMEM) were placed into the upper chambers. Cells were incubated at $37^{\circ} \mathrm{C}$ for 48 hours, then the inserts were removed, washed in ice-cold PBS, and noninvading cells together with extracellular matrix were removed from the upper surface of the filter by wiping with a cotton bud. Cells on the lower surface of the inserts were fixed in $4 \%$ paraformaldehyde, stained with crystal violet, and observed using an inverted phase-contrast microscope (Leica). The number of cells that had invaded to the lower surface was counted at a magnification of $\times 200$. The results were assessed independently by two researchers, and the invasive index was calculated as the proportion of the invading cells in each experimental group expressed relative to control cells. Each experiment was repeated independently three times.

\section{Statistical Analysis}

Data were analyzed using the independent sample $t$-test or one-way analysis of variance followed by the Tukey test. Correlations were analyzed using the Spearman rank correlation test. Data are presented as means \pm SD. All $P$ values are two-sided. $P<0.05$ was considered statistically significant. All statistical calculations were performed using SPSS 16.0 (Chicago, IL).

\section{Results}

STMN1 Is Down-Regulated in Cytotrophoblasts from Patients with RM

Quantitative real-time PCR and Western blot analysis of first-trimester chorionic villi tissue were performed to explore whether STMN1 is involved in the pathogenesis of RM. STMN1 expression was down-regulated significantly in the villi tissue of patients with RM (Figure 1, A and B). Immunohistochemical analysis of paraffin-embedded tissue was performed to investigate STMN1 localization further in first-trimester villi. Stronger expression of STMN1 was observed in $\mathrm{HC}$ villi tissue, with the positive cells mainly being cytotrophoblasts, although no positive signal was found in syncytiotrophoblasts. In addition, no positive signal for STMN1 was detected in villi tissue from patients with RM (Figure 1, C and D). These findings were confirmed by Western blot analysis, which showed that STMN1 was expressed at a higher level in HCs than patients with RM (Figure 1, E and F). Taken together, these findings

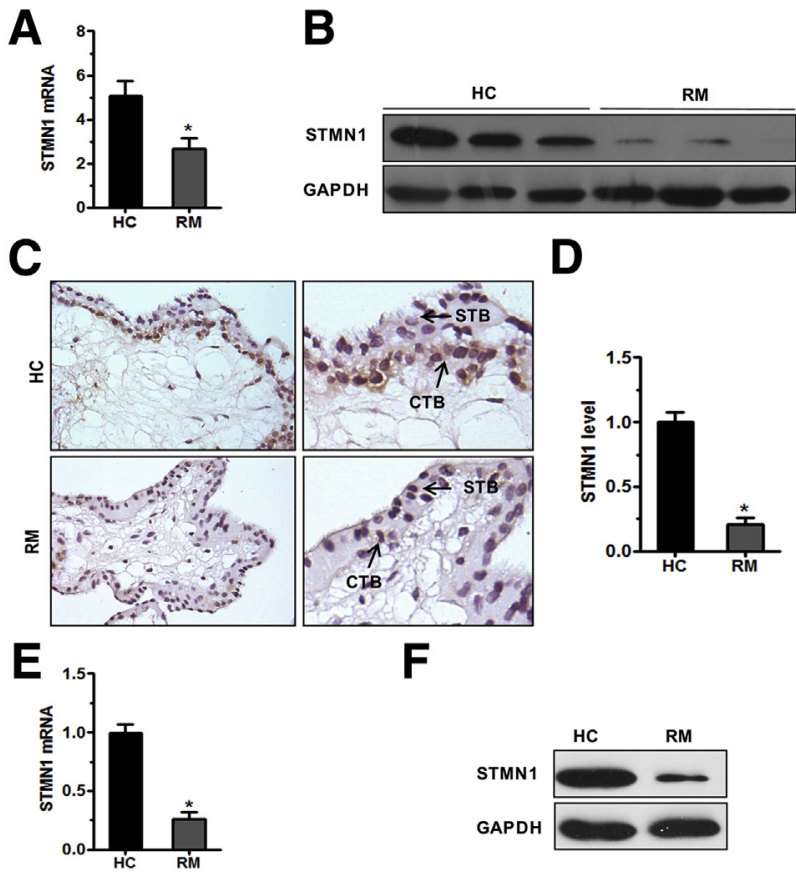

Figure 1 Stathmin-1 (STMN1) is down-regulated in first-trimester placental cytotrophoblasts in patients with recurrent miscarriage (RM). A and B: STMN1 expression in first-trimester human villi tissues from patients with RM or in healthy controls (HCs) was determined by quantitative RT-PCR (RT-qPCR) and Western blot analysis. $\mathbf{C}$ and $\mathbf{D}$ : Single staining of sections of maternal villi [cytotrophoblasts (CTB) and syncytiotrophoblasts (STB)] using rabbit IgG antihuman-STMN1 and developed using the streptavidin biotin plus horseradish peroxidase kit (Boster, Wuhan, China). The sections were counterstained with hematoxylin, and positive cells were quantified using ImagePro-plus 6.0. E and F: Levels of STMN1 expressed in cytotrophoblasts isolated from first-trimester human villi tissue from patients with RM or in HCs, as analyzed by RT-qPCR and Western blot analysis. $n=6(\mathbf{A}$ and $\mathbf{B}) ; n=14$ (C and D). ${ }^{*} P<0.05$ versus HCs. Original magnification: $\times 40$ (left); $\times 100$ (right). GAPDH, glyceraldehyde-3-phosphate dehydrogenase. 

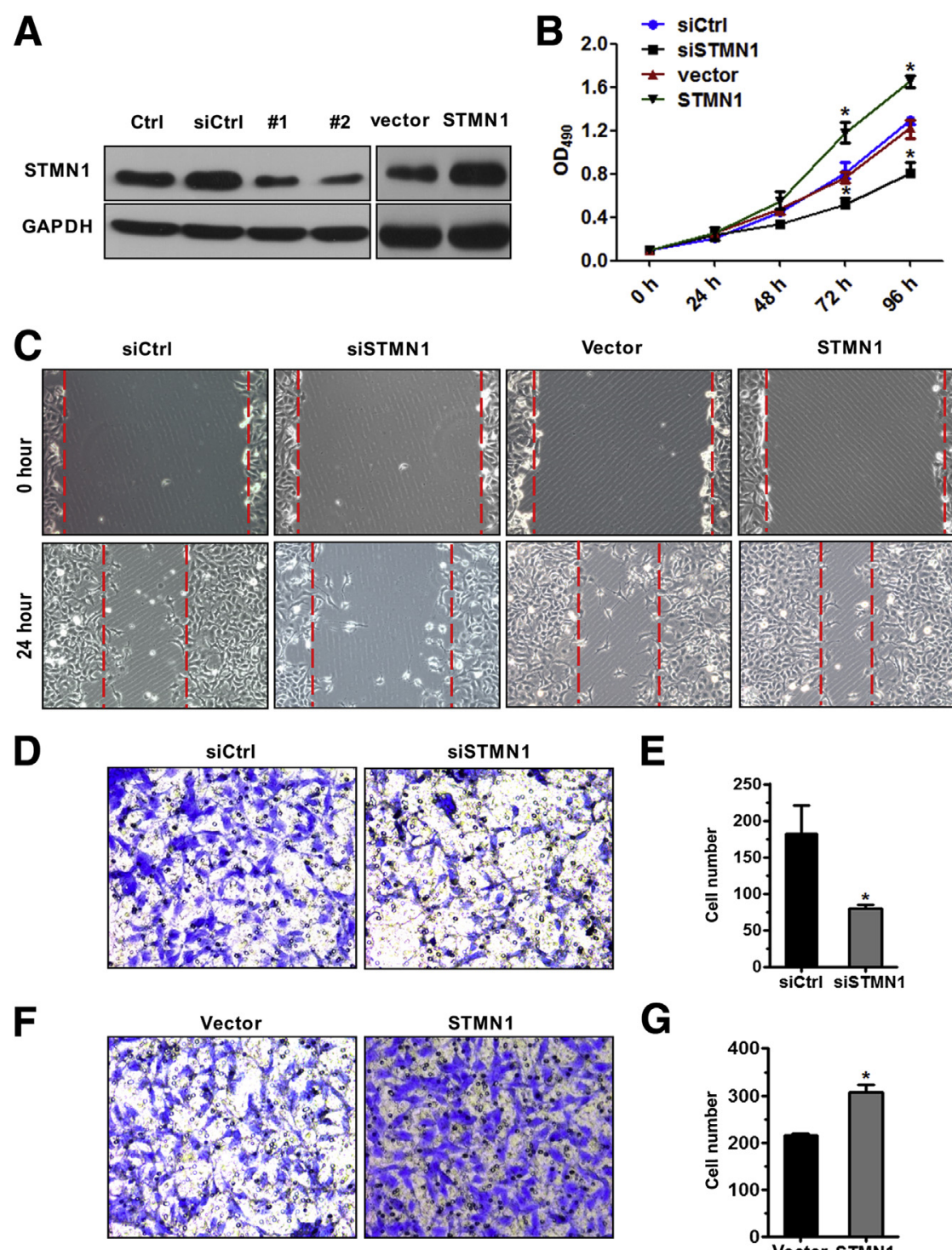

G

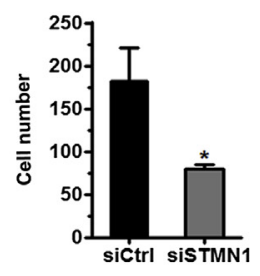

Figure 2 Knockdown of STMN1 decreases trophoblast proliferation and migration. A: Western blot analysis of stathmin-1 (STMN1) expression in HTR-8 cells transfected with small interfering Ctrl (siCtrl), siSTMN1 1, siSTMN1 2 oligonucleotides, control vector, or the STMN1-overexpressing vector after 48 hours. B: HTR-8 cells were transfected with siCtrl, siSTMN1 oligos, control vector, or the STMN1-overexpressing vector for 48 hours. Cell proliferation was measured after 48 hours using the MTT assay. C: Overexpression of STMN1 in HTR-8 cells results in increased wound closure ability compared with the vector control cells. Knockdown of STMN1 drastically reduced the rate of wound closure in comparison with the scrambled control cell line. D-G: Overexpression of STMN1 in HTR-8 cells significantly increases invasion compared with the vector control cell line. Knockdown of STMN1 reduces invasion compared with the scrambled control cell line. B, E, and G: ${ }^{*} P<0.05$ versus siCtrl or control vector. Original magnification: $\times 100(\mathbf{C}) ; \times 200($ D-G). GAPDH, glyceraldehyde-3-phosphate dehydrogenase. indicate that STMN1 expression in cytotrophoblasts is down-regulated significantly in patients with RM, suggesting that STMN1 may correlate with cytotrophoblast proliferation and invasion.

\section{STMN1 Regulates Trophoblast Proliferation and Invasion in Vitro}

To determine the effects of STMN1 on trophoblasts, HTR-8/ SVneo (HTR-8) cell line, a first-trimester human extravillous cytotrophoblast-derived cell line, ${ }^{22}$ was transfected with siSTMN1 oligonucleotides or the STMN1-overexpressing vector. STMN1 expression decreased after siSTMN1 transfection, but was up-regulated after transfection of the STMN1overexpressing vector (Figure 2A). The MTT assay and a fluorescence-activated cell sorter showed that overexpression of STMN1 increased HTR-8 cell proliferation, whereas knockdown of STMN1 decreased HTR-8 cell proliferation (Figure 2B and Supplemental Figure S1). Furthermore, the wound healing assay and Matrigel invasion assay showed that overexpression of STMN1 significantly increased the migratory and invasive ability of HTR-8 cells, whereas knockdown of STMN1 significantly reduced migration and invasion (Figure 2, C-G). These results suggest that STMN1 may play a key role in trophoblast proliferation, migration, and invasion.

In trophoblasts, cadherins regulate the balance between suppression and promotion of invasion: E-cadherin suppresses invasion whereas $\mathrm{N}$-cadherin promotes invasion. $^{25,26}$ To explore whether STMN1 promotes the epithelial-mesenchymal transition in trophoblasts by regulating E-cadherin and N-cadherin, we assessed STMN1, E-cadherin, and N-cadherin expression in HTR-8 cells using an immunofluorescent assay. Increased immunoreactivity for E-cadherin was detected in the cytoplasm of HTR-8 cells after knockdown of STMN1. In contrast, N-cadherin and STMN1 immunoreactivity obviously was reduced after 
A

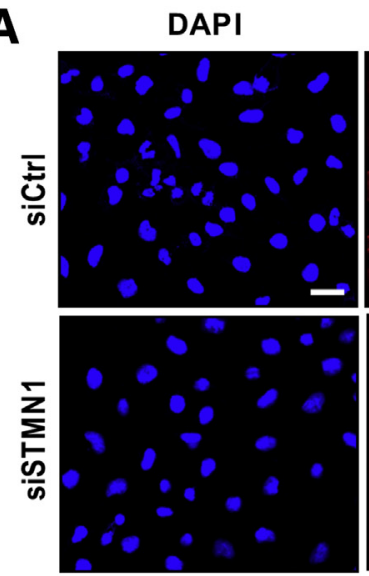

C

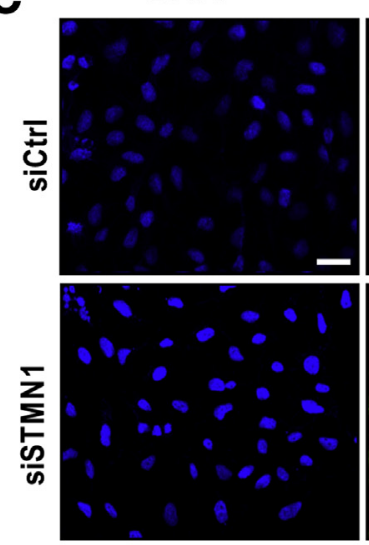

E

픔

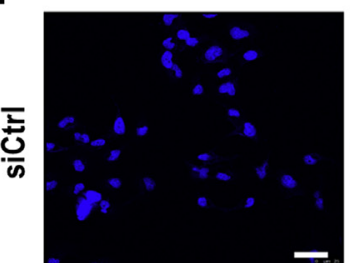

$\sum_{\frac{C}{6}}^{\frac{5}{6}}$

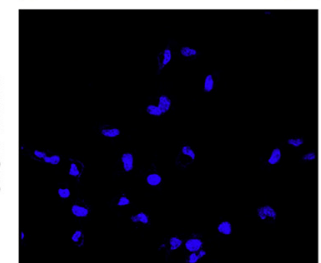

STMN1

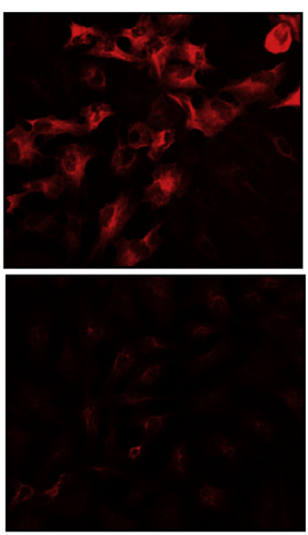

E-cadherin
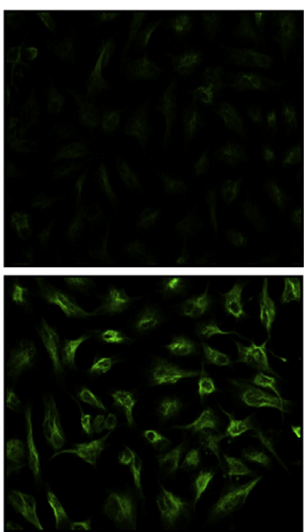

$\mathbf{N}$-cadherin
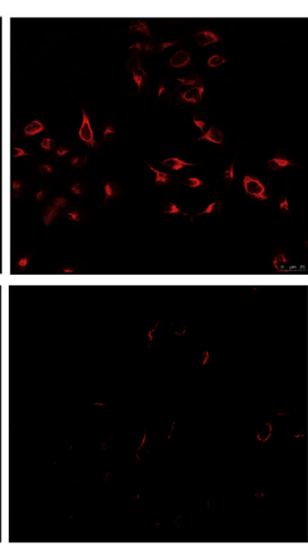

Merge

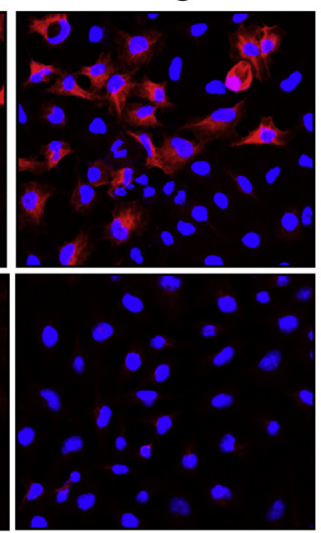

Merge

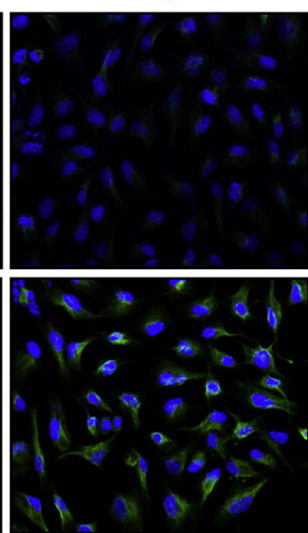

Merge
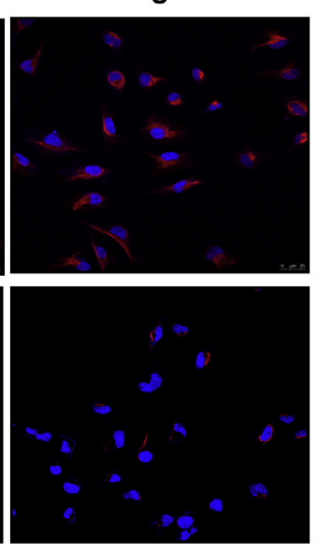

B

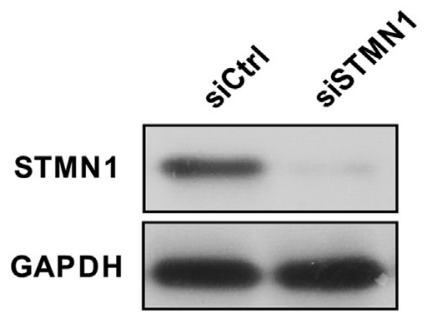

D

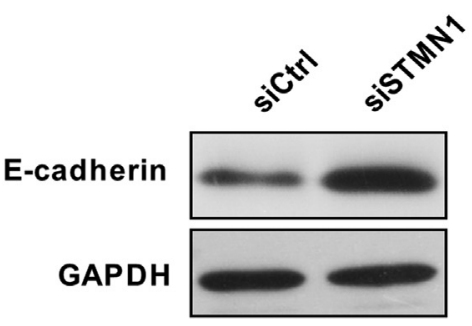

F

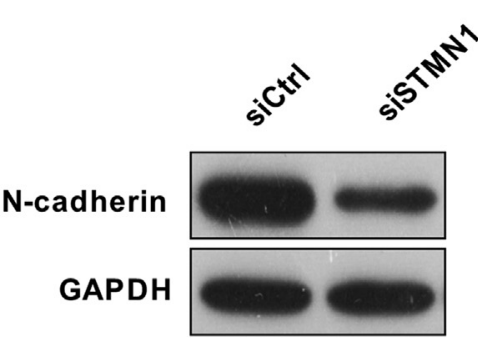

Figure 3 Effects of knocking down STMN1 on N-cadherin and E-cadherin expression in HTR-8 cells. HTR-8 cells derived from human invasive extravillous trophoblasts were transfected with small interfering Ctrl (siCtrl) or small interfering stathmin-1 (siSTMN1) oligos. STMN1 (A and B), E-cadherin (C and D), and $\mathrm{N}$-cadherin (E and $\mathbf{F})$ protein expression were assessed 48 hours later by confocal imaging $(\mathbf{A}, \mathbf{C}$, and $\mathbf{E})$ and immunoblotting $(\mathbf{B}, \mathbf{D}$, and $\mathbf{F})$. Scale bars: $25 \mu \mathrm{m}$. GAPDH, glyceraldehyde-3-phosphate dehydrogenase.

knockdown of STMN1 (Figure 3, A, C, and E). Western blot analysis confirmed that knockdown STMN1 decreased Ncadherin expression but increased E-cadherin expression in HTR-8 cells (Figure 3, B, D, and F).

Furthermore, immunohistochemical staining for E-cadherin and $\mathrm{N}$-cadherin in human first-trimester villi tissue showed that E-cadherin was distributed mainly in cytotrophoblasts, and was expressed at higher levels in patients with RM (Figure 4, A and B). Conversely, N-cadherin was down-regulated in the first-trimester villi tissue of patients with RM (Figure 4, C and D). Collectively, these findings suggest that STMN1 promotes the migration and invasion of trophoblasts by regulating the protein expression of E-cadherin and N-cadherin. 
Reduced STMN1 Expression Impairs Nucleocytoplasmic Translocation of $\beta$-Catenin and Decreases Trophoblast Invasion

Previous studies have reported that the E-cadherin $/ \beta$-catenin complex plays a key role in maintaining epithelial integrity and regulating the translocation of cytoplasmic $\beta$-catenin into the nucleus. ${ }^{27,28}$ To gain further insight into the molecular events underlying the ability of STMN1 to regulate trophoblast migration and invasion, HTR- 8 cells were transfected with STMN1 siRNA or control siRNA for 48 hours. Confocal imaging showed that $\beta$-catenin was distributed mainly in the nuclei of control-transfected HTR8 cells, whereas knockdown of STMN1 obviously reduced nuclear accumulation of $\beta$-catenin (Figure 5A). Nuclear/ cytoplasmic fractionation and Western blot analysis confirmed that significant amounts of $\beta$-catenin could be detected in the cytoplasm of HTR-8 cells after knockdown of STMN1 (Figure 5B).

Numerous studies have reported that MMPs, which are regulated mainly by the $\mathrm{Wnt} / \beta$-catenin pathway, play a key role in trophoblast invasion. ${ }^{21}$ MMPs are required to create a microenvironment that enables trophoblast migration and invasion. Therefore, to further determine the effect of STMN1 on trophoblast invasion and migration, we used antibody array technology to determine the expression profiles of 10 human MMP-related proteins in the supernatant of HTR-8 cells after knockdown of STMN1. MMP-1, MMP-2, MMP-8, and MMP-9 expression was downregulated significantly after knockdown of STMN1, whereas expression of TIMP1 and TIMP2 obviously increased and the levels of TIMP4, MMP-3, MMP-10, and MMP-13 expression were not affected significantly (Figure 6, A-L). These results suggest that STMN1 regulates the migration and invasion of trophoblasts via the E-cadherin/ $\beta$-catenin pathway.

\section{TNF- $\alpha$ Represses STMN1 Expression in Trophoblasts}

A previous study reported that abnormal levels of various cytokines, especially IFN- $\gamma$ and TNF- $\alpha$, inhibited trophoblast growth and invasion in patients with RM. ${ }^{29}$ To investigate whether these factors also are involved in the regulation of STMN1 expression in trophoblasts, primary trophoblasts were treated with LPS, IFN- $\gamma$, or TNF- $\alpha$ for 24 hours. STMN1 expression was down-regulated significantly in trophoblasts treated with TNF- $\alpha$, but obviously was not altered in cells treated with IFN- $\gamma$ or LPS. Further analysis showed that STMN1 expression was down-regulated more significantly in TNF- $\alpha$-induced trophoblasts than in trophoblasts treated with IFN- $\gamma$ plus TNF- $\alpha$ (Figure 7, A and B). Serum TNF- $\alpha$ was significantly lower in patients with
A

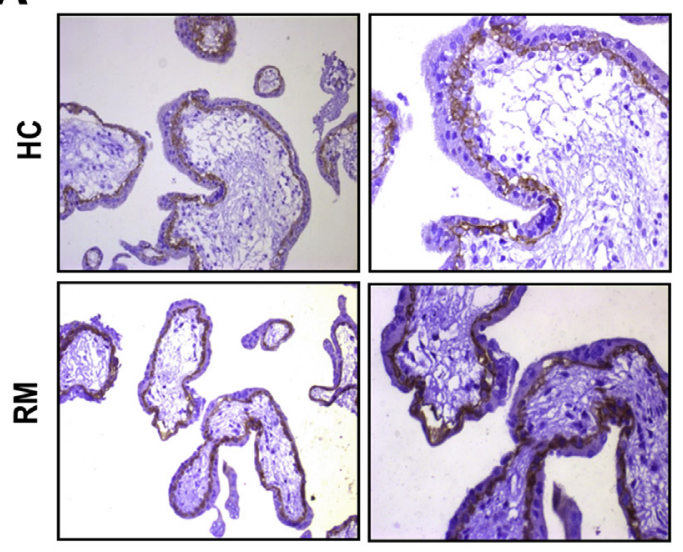

C

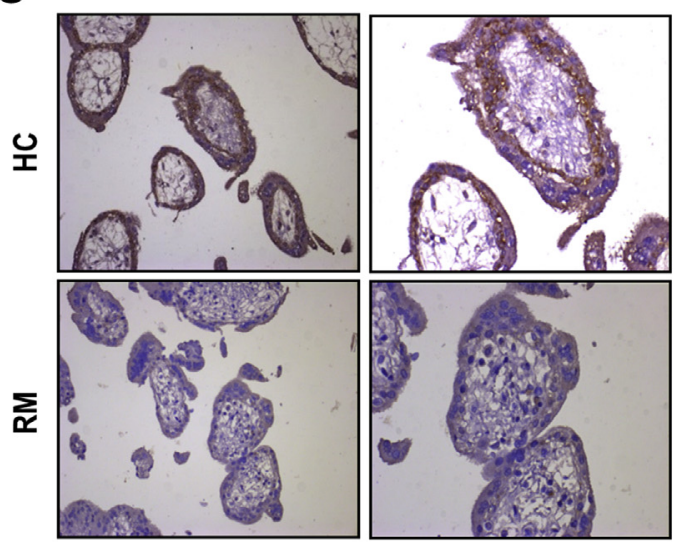

B

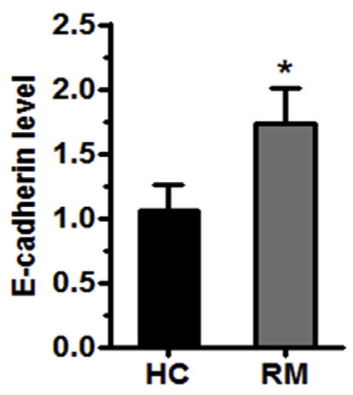

D

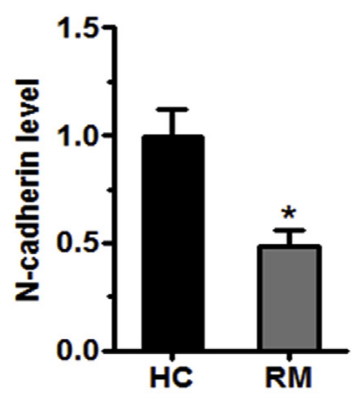

Figure 4 Expression of $\mathrm{N}$-cadherin and $\mathrm{E}-$ cadherin in the placental villi of patients with recurrent miscarriage (RM). Villi sections from human healthy controls ( $\mathrm{HCs}$ ) or patients with RM were subjected to single staining using rabbit IgG human E-cadherin (A and $\mathbf{B}$ ) or $\mathrm{N}$-cadherin antibodies (C and $\mathbf{D})$, developed using the labeled streptavidin biotin plus horseradish-peroxidase kit (Boster), counterstained with hematoxylin, and positive cells were quantified using ImagePro-plus 6.0 software (Media Cybernetics, Rockville, MD; B and D). $n=14$. ${ }^{*} P<0.05$ versus $\mathrm{HCs}$. $\mathbf{A}$ and $\mathbf{C}$ : Original magnification: $\times 40$ (left); $\times 100$ (right). 

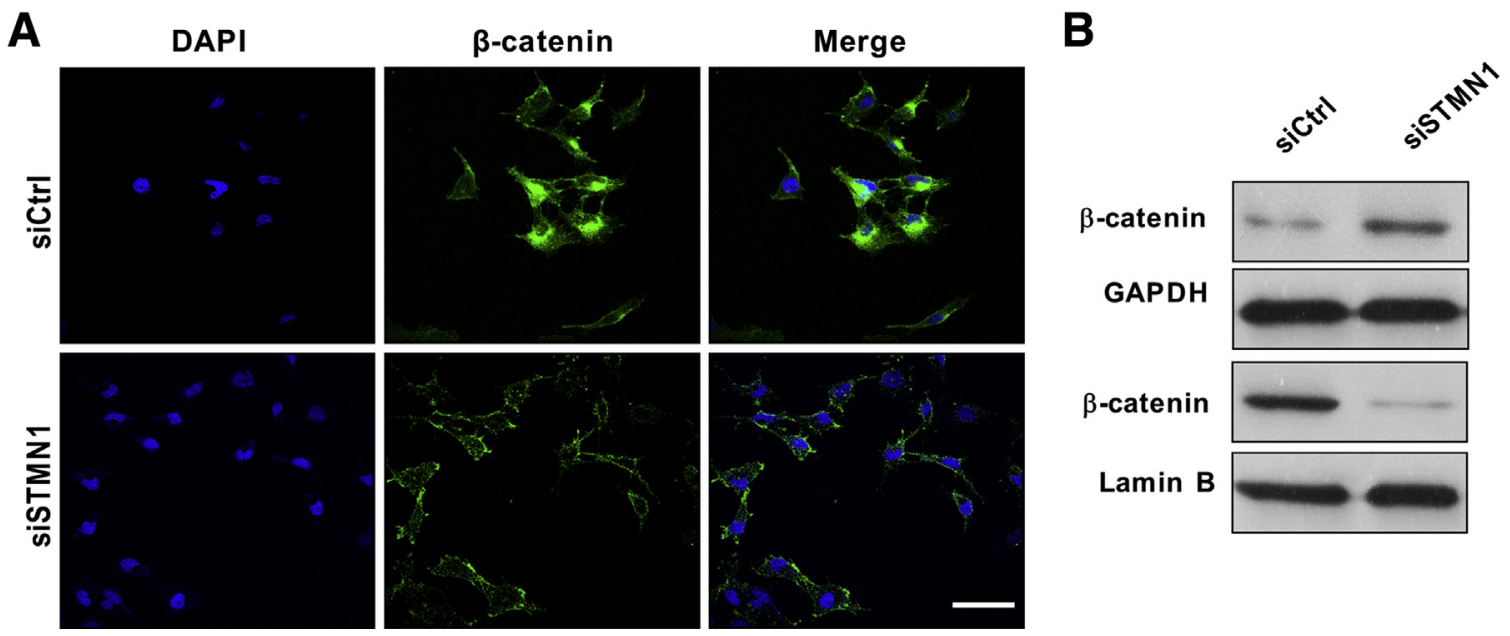

Figure 5 Knockdown of STMN1 attenuates nuclear translocation of $\beta$-catenin in HTR-8 cells. HTR-8 cells were transfected with small interfering Ctrl (siCtrl) or small interfering stathmin-1 (siSTMN1) oligos, and cultured for 48 hours. The cells were subjected to immunofluorescent staining for $\beta$-catenin (green) and counterstained with DAPI (blue) to assess the subcellular localization of $\beta$-catenin (A) and immunoblotting to compare the levels of $\beta$-catenin in the nucleus and cytoplasm (B). Scale bar $=25 \mu \mathrm{m}$ (A). GAPDH, glyceraldehyde-3-phosphate dehydrogenase.

RM than in HCs (Figure 7C). We also detected TNF- $\alpha$ expression at 48 hours in HTR-8 cells that had been transfected with a control vector or STMN1-overexpressing plasmid. No significant change was observed in TNF- $\alpha$ expression in STMN1-expressing cells in quantitative RTPCR analysis (Supplemental Figure S2). In addition, STMN1 mRNA expression in RM villi tissue correlated negatively with the serum level of TNF- $\alpha$ (Figure 7D). These results indicate that STMN1 expression in trophoblasts is regulated by TNF- $\alpha$, and that STMN1 expression in trophoblasts correlates inversely with the circulating levels of TNF- $\alpha$ in patients with RM.

\section{M1 Macrophage-Secreted TNF- $\alpha$ Reduces STMN1 Expression in Trophoblasts}

We previously reported that increased TNF- $\alpha$ induced pregnancy loss in nonobese diabetic mice, and depletion of macrophages using an F4/80 antibody abrogated TNF- $\alpha$ production and improved pregnancy outcomes in nonobese diabetic mice. ${ }^{30}$ To explore the ability of humanderived, macrophage-secreted TNF- $\alpha$ to repress STMN1 expression in trophoblasts, $\mathrm{CD} 14^{+}$monocytes were isolated from human umbilical cord blood and induced to obtained unactivated macrophages (M0), and then differentiated further to generate M1, M2a, M2b, or M2c macrophages. The cell culture supernatants were collected during both macrophage differentiation (denoted M0) and after differentiation (denoted M1, M2a, M2b, and M2c). TNF- $\alpha$ levels in the supernatants of the differentiated macrophages were assessed by enzyme-linked immunosorbent assay (Supplemental Figure S3). Primary trophoblasts were treated with the cell culture supernatants for 24 hours. Immunoblotting and RT-qPCR showed that the supernatant of M1 macrophages significantly inhibited
STMN1 expression in primary trophoblasts compared with macrophages cultured with the supernatant of M0 macrophages. However, trophoblast STMN1 expression was not altered significantly by treatment with the supernatants from M2a, M2b, or M2c macrophages (Figure 8, A and B).

To further confirm the role of the TNF- $\alpha /$ STMN1 axis on the interaction between macrophages and trophoblasts, we assessed the invasive ability of trophoblasts in a macrophage-trophoblast co-culture model. Compared with co-culture with M0 macrophages, co-culture with M1 macrophages significantly reduced trophoblast invasion. Moreover, the invasive indices of the TNF- $\alpha$-treated group and M1 macrophages were lower than that of unstimulated M0 macrophages (Figure 8, C and D). Interestingly, when TNF- $\alpha$ neutralizing antibody was added, the reduced ability of M1 trophoblasts to migrate was rescued (compared with the macrophage and trophoblast co-culture group) (Figure 8, E-H). These results strongly imply that M1 macrophage-secreted TNF- $\alpha$ may be the dominant paracrine regulator of the biological functions of trophoblasts in patients with RM.

\section{Discussion}

The establishment of a successful pregnancy requires trophoblast migration and invasion at the early stage of placentation. ${ }^{31,32}$ This study shows that knockdown of STMN1 significantly suppresses trophoblast proliferation and invasion. Further investigation showed that STMN1 directly regulates $\beta$-catenin translocation into the nucleus, which reduces MMP protein expression and inhibits trophoblast migration and invasion, and that M1 macrophage-secreted TNF- $\alpha$ negatively regulates STMN1 and N-cadherin expression in trophoblasts (Supplemental Figure S4). 
A

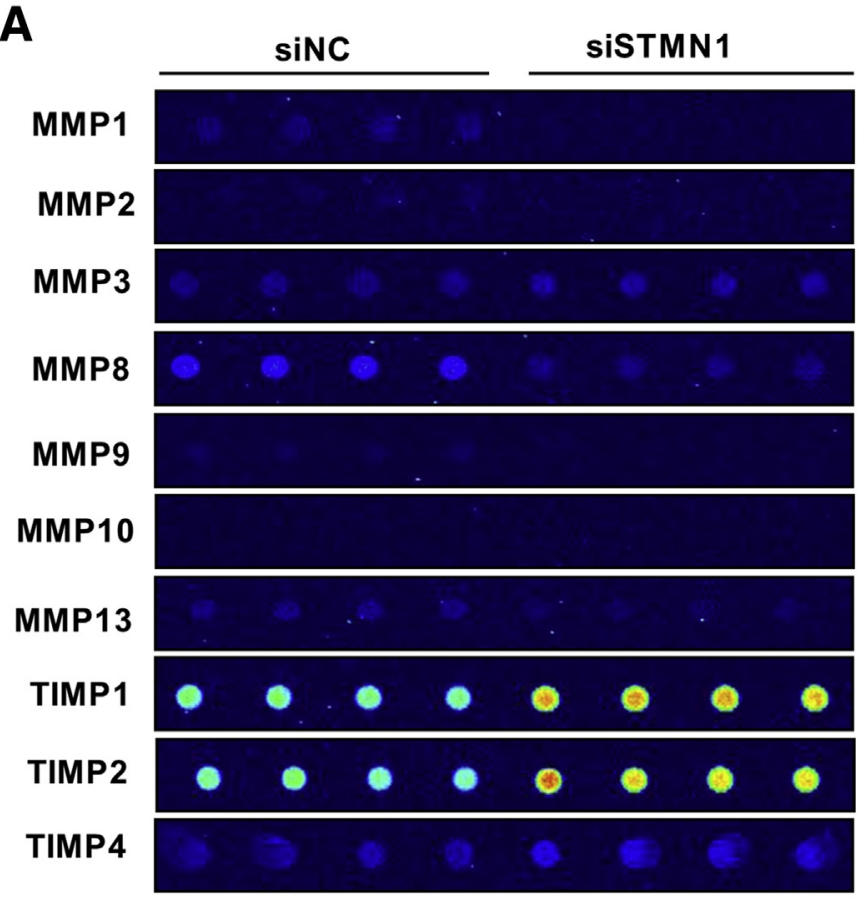

B

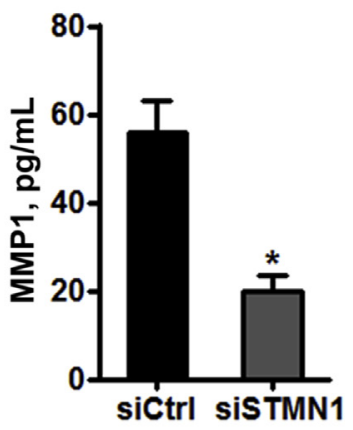

F

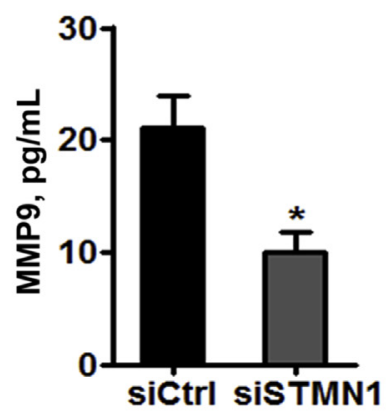

$\mathbf{J}$

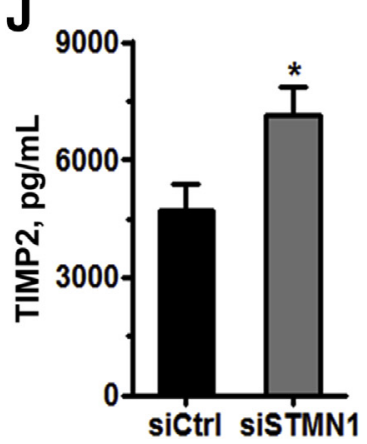

C

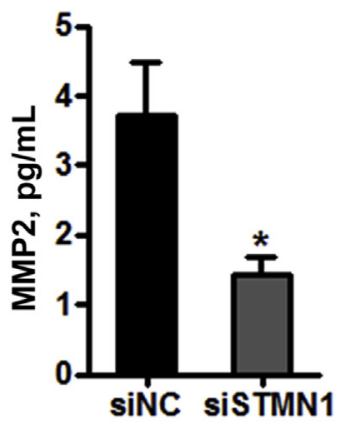

G

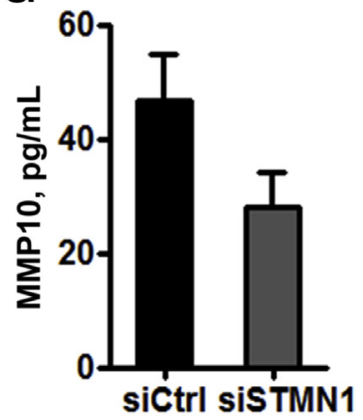

$\mathrm{K}$

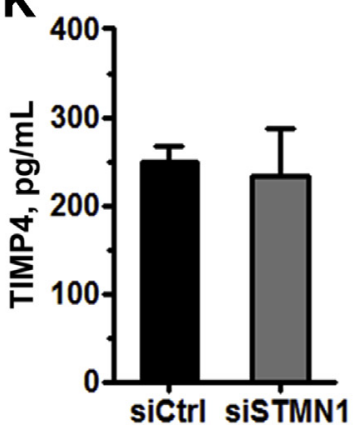

Figure 6 Knockdown of STMN1 decreases matrix metalloprotease (MMP) expression in trophoblasts. A-K: HTR-8 cells were transfected with small interfering Ctrl (siCtrl) or small interfering stathmin-1 (siSTMN1) oligos, cultured for 48 hours, and the levels of MMPs and TIMPs in the cell supernatant were quantified using the Quantibody Human MMP Array 1 (Norcross, GA). ${ }^{*} P<0.05$ versus siCtrl.

Interestingly, we also showed that STMN1 is downregulated in first-trimester villi tissue and cytotrophoblasts in patients with RM. Our previous study suggested that STMN1 expression was decreased significantly in uterine natural killer cells from inbred mouse strains CBA/ $\mathrm{J} \times \mathrm{DBA} / 2 \mathrm{~J}$ matings as compared with $\mathrm{CBA} / \mathrm{J} \times \mathrm{BALB} / \mathrm{c}$ matings. ${ }^{23}$ A neutralizing antibody against STMN1 further increased the percentage of embryo loss in CBA/J $\times$ DBA $/ 2 \mathrm{~J}$ matings. A similar trend was observed in the frequency of STMN $-1^{+} / \mathrm{CD} 6^{+}$cells in patients with RM and in HCs. ${ }^{23}$

During pregnancy, trophoblast invasion and uterine spiral artery remodeling are required to decrease maternal vascular resistance and increase uteroplacental blood flow, and impaired spiral artery remodeling is believed to be 


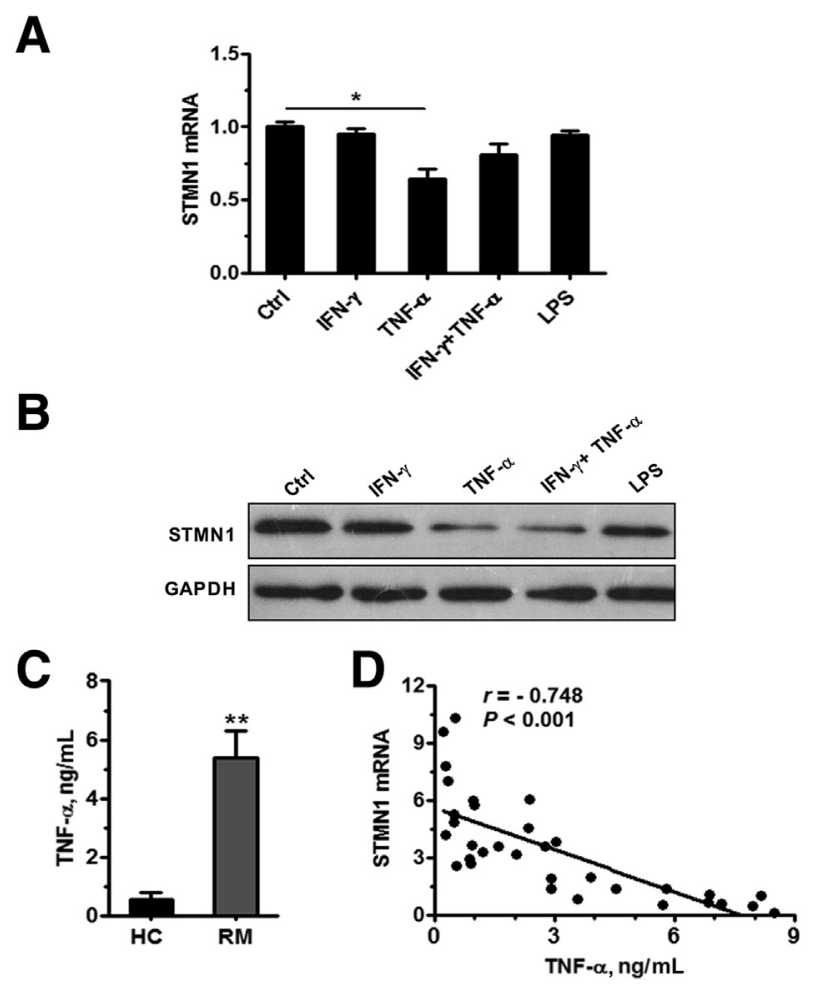

Figure 7 Tumor necrosis factor (TNF)- $\alpha$ reduces STMN1 expression in trophoblasts. Immunoblotting (A) and quantitative RT-PCR (RT-qPCR) (B) analysis of stathmin-1 (STMN1) expression in primary trophoblasts treated with interferon (IFN) $-\gamma$, TNF- $\alpha$, IFN- $\gamma+$ TNF- $\alpha$, or lipopolysaccharide (LPS) for 24 hours. C: Serum TNF- $\alpha$ levels of patients with recurrent miscarriage (RM) were determined using an enzyme-linked immunosorbent assay. D: STMN1 mRNA expression in primary trophoblasts (as determined by RT-qPCR analysis) correlated inversely with the serum TNF- $\alpha$ level in patients with RM. ${ }^{*} P<0.05$ versus controls, ${ }^{*} P<0.05$ versus healthy controls. Ctrl, control; $\mathrm{GAPDH}$, glyceraldehyde-3-phosphate dehydrogenase; HC, healthy control.

implicated in PE. ${ }^{3,33}$ In this study, immunohistochemical staining for STMN1 was performed in first-trimester villi tissue from $\mathrm{HCs}$ and in third-trimester placenta tissue from patients with $\mathrm{PE}$ and in HCs. A robust expression of STMN1 was observed in cytotrophoblasts from firsttrimester $\mathrm{HC}$ villi tissue, whereas no STMN1 signal was found in the indicated PE or $\mathrm{HC}$ placental tissue (Supplemental Figure S5). Taken together, these results suggest that sufficient STMN1 expression at the maternalfetal interface may be important for modulating uterine natural killer cell and trophoblast function and may be beneficial for pregnancy, at least at earlier stages of gestation. Whether impaired STMN1 expression is involved in the occurrence of PE is worthy of investigation in further research.

During early pregnancy, embryonic trophoblasts proliferate and differentiate into tumor-like cells that invade the uterus and vasculature to remodel the maternal spiral arteries. ${ }^{31}$ Interestingly, STMN1 is expressed in the glandular epithelium and stromal cells of human endometrial tissue by cytotrophoblasts and extravillous trophoblasts, but not by syncytiotrophoblasts. When stromal cells isolated from normal endometrial tissue were cultured and stimulated by progesterone plus estrogen or cAMP, both total STMN1 and phosphorylated STMN1 levels decreased. In contrast, decidualization was suppressed markedly by silencing STMN1. This suggests that progesterone, estrogen, and cAMP may modulate STMN1 production and phosphorylation, and that STMN1 may play a key role in modulating decidualization. ${ }^{34}$

Recently, Yoshie et al ${ }^{35}$ reported that STMN1 expression is associated with early trophoblast migration and differentiation into syncytiotrophoblasts in vitro. However, the genes downstream of STMN1 involved in these processes remain to be identified. This study shows that STMN1 is down-regulated in trophoblasts from patients with RM as compared with HCs. In addition, gain-of-function and loss-of-function analyses showed that STMN1 regulates trophoblast proliferation and invasion in vitro. Further study showed that knockdown of STMN1 in HTR-8 cells significantly promoted E-cadherin expression and inhibited N-cadherin expression. Previous findings showed that the E-cadherin/ $\beta$-catenin complex maintains the integrity of epithelial cell-cell contact, and loss of E-cadherin-mediated cell adhesion can promote $\beta$-catenin signaling. ${ }^{36}$ Our data showed that knockdown of STMN1 in HTR-8 cells decreased nuclear accumulation of $\beta$-catenin. These results suggest that the E-cadherin/ $\beta$-catenin pathway may act downstream of STMN1 to regulate trophoblast proliferation, migration, and invasion.

During embryo implantation, extravillous trophoblasts degrade extracellular matrix and invade into the maternal decidua and myometrium. ${ }^{37}$ The invasive potential of trophoblast correlates closely with their ability to secrete a variety of proteases, in particular those of the MMP family. ${ }^{38}$ MMP-2 and MMP-9 are expressed by human firsttrimester cytotrophoblasts, and overexpression of MMP-9 correlates positively with trophoblast invasion. ${ }^{39}$ By using a human MMP array, we observed that knockdown of STMN1 inhibited MMP-2 and MMP-9 expression in trophoblasts. Thus, these findings suggest that STMN1 contributes to trophoblast migration and invasion by regulating MMP-2 and MMP-9 expression.

Decidual macrophages comprise the second largest decidual leukocyte population in early pregnancy. ${ }^{40}$ However, their precise function has not been defined. Various studies in model systems have categorized macrophages into different subtypes based on their cell surface phenotype, cytokine production, and functional properties. ${ }^{41}$ Macrophage phenotypes generally are divided into the major subsets M1 and M2 (M2a, M2b, and M2c). ${ }^{42,43}$ In normal pregnancy, M2 macrophages, which exist mainly in decidua tissue during early pregnancy, produce immunomodulatory proteins such as transforming growth factor- $\beta$ and IL-10 to promote fetaltrophoblast invasion and migration. ${ }^{10,44}$ However, in patients with RM, M2 macrophages transform into M1 macrophages that are capable of producing proinflammatory cytokines, such as TNF- $\alpha$ and IL- $1 \beta .{ }^{45}$ Interestingly, our data showed that M1 macrophage-derived TNF- $\alpha$ effectively inhibited STMN1 expression in trophoblasts, and in a macrophage-trophoblast 


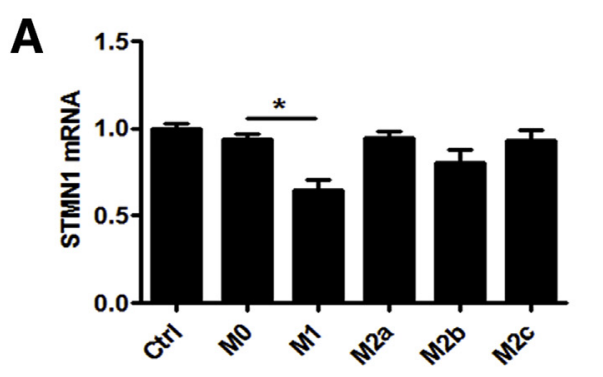

C

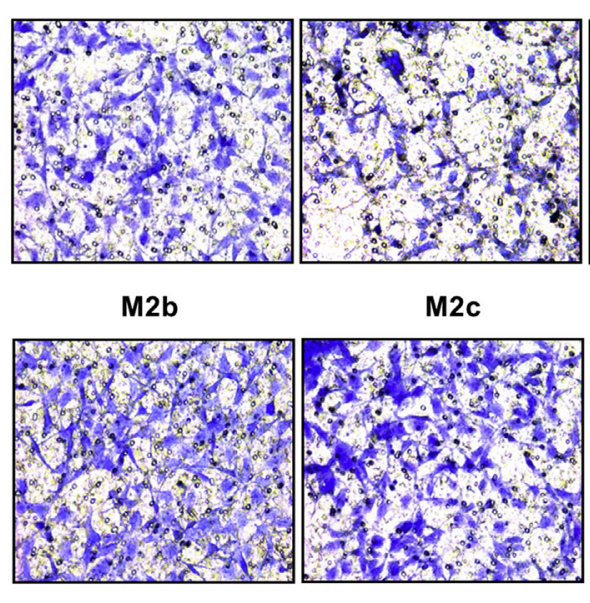

E

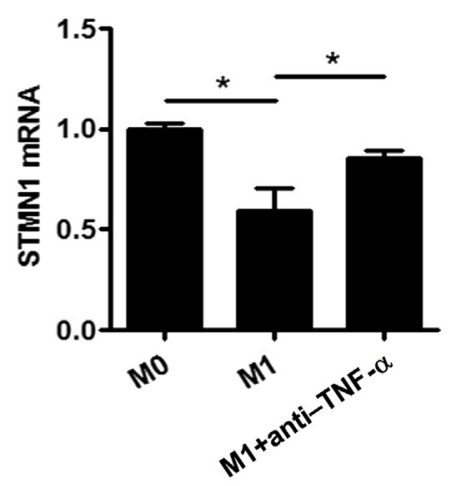

G

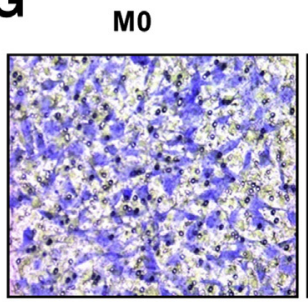

B

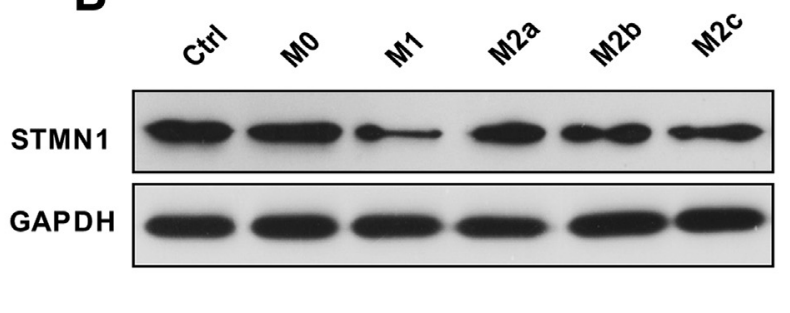

M2a
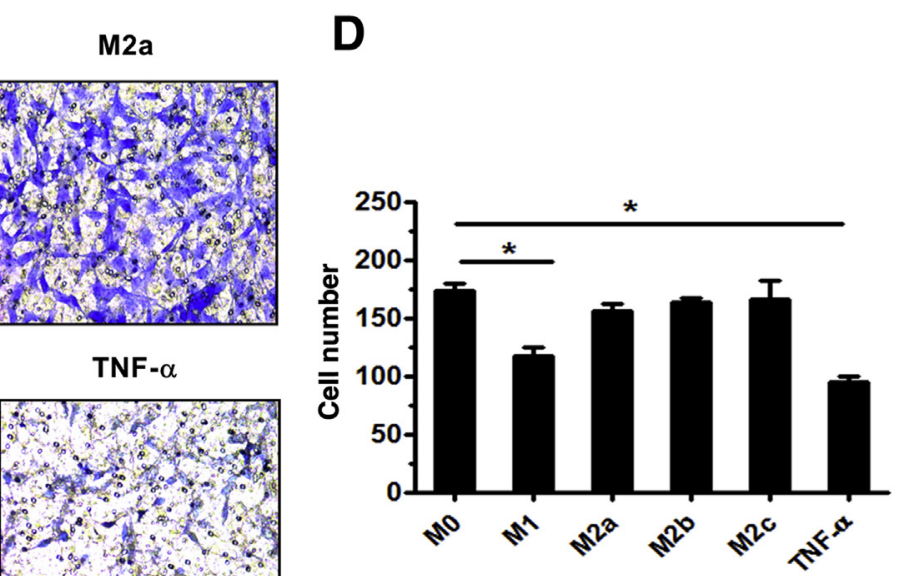

TNF $-\alpha$

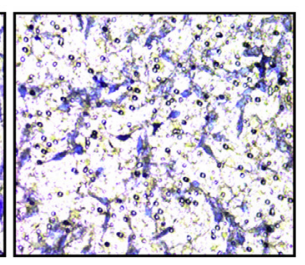

$\mathbf{F}$

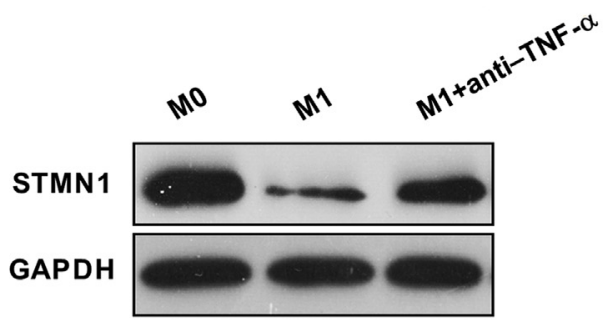

H

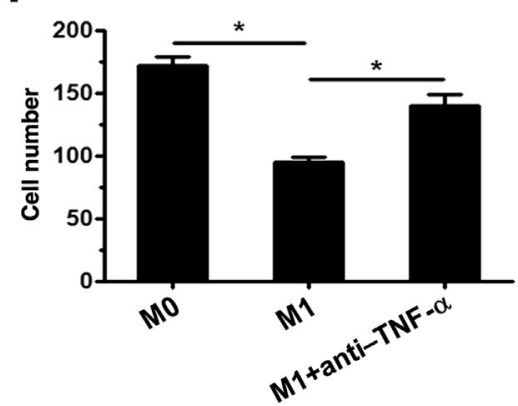

Figure 8 M1 macrophage-derived tumor necrosis factor (TNF)- $\alpha$ inhibits trophoblast migration and invasion. M0 macrophages were differentiated into M1, M2a, $M 2 b$, and M2c macrophages, and cell supernatants were harvested. Quantitative RT-PCR (RT-qPCR; A) and immunoblotting (B) analysis of stathmin-1 (STMN1) expression in trophoblasts treated with the supernatants of differentiated macrophages for 24 hours. $\mathbf{C}$ and $\mathbf{D}$ : A co-culture system was established to evaluate the invasive ability of trophoblasts after culture with differentiated macrophages. Differentiated macrophages or TNF- $\alpha$ were seeded into the lower chamber and trophoblasts were seeded into the upper chamber of Transwell inserts (Corning). The average number of invading cells per field was determined and compared with the MO macrophage group. E and $\mathbf{F}$ : RT-qPCR and immunoblotting analysis of STMN1 expression in trophoblast cells treated for 24 hours with the cell supernatants of M0 macrophages, M1 macrophages, or M1 macrophages + anti-TNF- $\alpha . \mathbf{G}$ and H: M0 macrophages, M1 macrophages, or M1 macrophages + anti-TNF- $\alpha$ (lower layer) and trophoblast cells (upper layer) were seeded into the lower and upper layers of Transwell inserts. The average number of invading cells per field was determined and compared with $M 0$ macrophages. ${ }^{\star} P<0.05$ versus M0 macrophages. Original magnification: $\times 200$ (C, D, G, and H). Ctrl, control; GAPDH, glyceraldehyde-3-phosphate dehydrogenase. 
co-culture system, M1 macrophages obviously inhibited trophoblast invasion by regulating STMN1.

To clarify whether LPS regulates STMN1 expression in trophoblasts, primary trophoblasts and HTR-8 cells were treated with different concentrations of LPS for varying times. However, no significant change was observed in the level of STMN1 expressed (Supplemental Figure S6). These results suggest that LPS may not be a direct regulatory factor for STMN1 expression in trophoblasts. A previous report also indicated that, in co-culture experiments, human LPS-activated macrophages inhibited the ability of immortalized HTR-8/SVneo human trophoblast cells to invade by releasing TNF- $\alpha \cdot{ }^{46}$ In our current study, differential STMN1 expression was found on TNF- $\alpha$ stimulation (Figure 7, A, $\mathrm{B}$, and D), whereas no such effect was observed after LPS stimulation (Figure 7, A and B); however, the reason remains unclear. It is possible that LPS indirectly may inhibit STMN1 expression in trophoblasts, whereas TNF- $\alpha$ may inhibit STMN1 directly under certain conditions.

In summary, this study provides new insight into the unique trophoblast proliferation and invasion events that occur during early human pregnancy. Importantly, we provide evidence for transplacental regulation of cellular immunity and also of a previously unknown role for TNF- $\alpha$ in regulating trophoblast invasion. These novel findings have important implications for the role of trophoblast invasion in maintaining successful pregnancy.

\section{Acknowledgment}

The HTR-8/SVneo cell line was a generous gift from Dr. Peeyush K. Lala (University of Western Ontario, Ontario, Canada).

\section{Supplemental Data}

Supplemental material for this article can be found at http://dx.doi.org/10.1016/j.ajpath.2015.06.010.

\section{References}

1. Erlebacher A: Immunology of the maternal-fetal interface. Annu Rev Immunol 2013, 31:387-411

2. Red-Horse K, Zhou Y, Genbacev O, Prakobphol A, Foulk R, McMaster M, Fisher SJ: Trophoblast differentiation during embryo implantation and formation of the maternal-fetal interface. J Clin Invest 2004, 114:744-754

3. Powe CE, Levine RJ, Karumanchi SA: Preeclampsia, a disease of the maternal endothelium: the role of antiangiogenic factors and implications for later cardiovascular disease. Circulation 2011, 123:2856-2869

4. Hill JA, Polgar K, Anderson DJ: T-helper 1-type immunity to trophoblast in women with recurrent spontaneous abortion. JAMA 1995, 273:1933-1936

5. Rai R, Regan L: Recurrent miscarriage. Lancet 2006, 368:601-611

6. Hemberger M, Hughes M, Cross JC: Trophoblast stem cells differentiate in vitro into invasive trophoblast giant cells. Dev Biol 2004 271:362-371
7. Mellor AL, Munn DH: Immunology at the maternal-fetal interface: lessons for T cell tolerance and suppression. Annu Rev Immunol 2000, $18: 367-391$

8. Du MR, Wang SC, Li DJ: The integrative roles of chemokines at the maternal-fetal interface in early pregnancy. Cell Mol Immunol 2014, $11: 438-448$

9. Heikkinen J, Mottonen M, Komi J, Alanen A, Lassila O: Phenotypic characterization of human decidual macrophages. Clin Exp Immunol 2003, 131:498-505

10. Houser BL, Tilburgs T, Hill J, Nicotra ML, Strominger JL: Two unique human decidual macrophage populations. J Immunol 2011, $186: 2633-2642$

11. Murray PJ, Wynn TA: Protective and pathogenic functions of macrophage subsets. Nat Rev Immunol 2011, 11:723-737

12. Gustafsson C, Mjosberg J, Matussek A, Geffers R, Matthiesen L, Berg G, Sharma S, Buer J, Ernerudh J: Gene expression profiling of human decidual macrophages: evidence for immunosuppressive phenotype. PLoS One 2008, 3:e2078

13. Guenther S, Vrekoussis T, Heublein S, Bayer B, Anz D, Knabl J, Navrozoglou I, Dian D, Friese K, Makrigiannakis A, Jeschke U: Decidual macrophages are significantly increased in spontaneous miscarriages and over-express FasL: a potential role for macrophages in trophoblast apoptosis. Int J Mol Sci 2012, 13: 9069-9080

14. Jourdain L, Curmi P, Sobel A, Pantaloni D, Carlier MF: Stathmin: a tubulin-sequestering protein which forms a ternary T2S complex with two tubulin molecules. Biochemistry 1997, 36:10817-10821

15. Clement MJ, Jourdain I, Lachkar S, Savarin P, Gigant B, Knossow M, Toma F, Sobel A, Curmi PA: N-terminal stathmin-like peptides bind tubulin and impede microtubule assembly. Biochemistry 2005, 44: 14616-14625

16. Rubin CI, Atweh GF: The role of stathmin in the regulation of the cell cycle. J Cell Biochem 2004, 93:242-250

17. Chen J, Abi-Daoud M, Wang A, Yang X, Zhang X, Feilotter HE, Tron VA: Stathmin 1 is a potential novel oncogene in melanoma. Oncogene 2013, 32:1330-1337

18. Watanabe A, Suzuki H, Yokobori T, Tsukagoshi M, Altan B, Kubo N Suzuki S, Araki K, Wada S, Kashiwabara K, Hosouchi Y, Kuwano H: Stathmin1 regulates p27 expression, proliferation and drug resistance, resulting in poor clinical prognosis in cholangiocarcinoma. Cancer Sci 2014, 105:690-696

19. Tamura K, Yoshie M, Nishi H, Osakabe Y, Isaka K, Hara T, Kogo H: Expression of stathmin in human uterus and decidualizing endometrial stromal cells. Reproduction 2006, 132:625-636

20. Rao AJ: Proteomic profiling of forskolin-induced differentiated BeWo cells: an in-vitro model of cytotrophoblast differentiation. Reprod Biomed Online 2007, 14:477-487

21. Zhou WH, Du MR, Dong L, Zhu XY, Yang JY, He YY, Li DJ: Cyclosporin A increases expression of matrix metalloproteinase 9 and 2 and invasiveness in vitro of the first-trimester human trophoblast cells via the mitogen-activated protein kinase pathway. Hum Reprod 2007, 22:2743-2750

22. Graham CH, Hawley TS, Hawley RG, MacDougall JR, Kerbel RS, Khoo N, Lala PK: Establishment and characterization of first trimester human trophoblast cells with extended lifespan. Exp Cell Res 1993 206:204-211

23. Lin Y, Li C, Shan B, Wang W, Saito S, Xu J, Di J, Zhong Y, Li DJ: Reduced stathmin-1 expression in natural killer cells associated with spontaneous abortion. Am J Pathol 2011, 178:506-514

24. Qiu Q, Yangi MY, Tsang BK, Gruslin A: Both mitogen-activated protein kinase and phosphatidylinositol 3-kinase signalling are required in epidermal growth factor-induced human trophoblast migration. Mol Hum Reprod 2004, 10:677-684

25. Lamouille S, Xu J, Derynck R: Molecular mechanisms of epithelialmesenchymal transition. Nat Rev Mol Cell Biol 2014, 15:178-196

26. Floridon C, Nielsen O, Holund B, Sunde L, Westergaard JG, Thomsen SG, Teisner B: Localization of E-cadherin in villous, 
extravillous and vascular trophoblasts during intrauterine, ectopic and molar pregnancy. Mol Hum Reprod 2000, 6:943-950

27. Conacci-Sorrell M, Zhurinsky J, Ben-Ze'ev A: The cadherin-catenin adhesion system in signaling and cancer. J Clin Invest 2002, 109: 987-991

28. Jeanes A, Gottardi CJ, Yap AS: Cadherins and cancer: how does cadherin dysfunction promote tumor progression? Oncogene 2008, 27 : 6920-6929

29. Laird SM, Tuckerman EM, Cork BA, Linjawi S, Blakemore AI, Li TC: A review of immune cells and molecules in women with recurrent miscarriage. Hum Reprod Update 2003, 9:163-174

30. Sun Y, Qin X, Shan B, Wang W, Zhu Q, Sharma S, Wu J, Lin Y: Differential effects of the CpG-Toll-like receptor 9 axis on pregnancy outcome in nonobese diabetic mice and wild-type controls. Fertil Steril 2013, 99:1759-1767

31. Burrows TD, King A, Loke YW: Trophoblast migration during human placental implantation. Hum Reprod Update 1996, 2:307-321

32. Colicchia M, Campagnolo L, Baldini E, Ulisse S, Valensise H, Moretti C: Molecular basis of thyrotropin and thyroid hormone action during implantation and early development. Hum Reprod Update 2014, 20:884-904

33. Cui Y, Wang W, Dong N, Lou J, Srinivasan DK, Cheng W, Huang X, Liu M, Fang C, Peng J, Chen S, Wu S, Liu Z, Dong L, Zhou Y, Wu Q: Role of corin in trophoblast invasion and uterine spiral artery remodelling in pregnancy. Nature 2012, 484:246-250

34. Gou J, Jia J, Zhao X, Yi T, Li Z: Identification of stathmin 1 during peri-implantation period in mouse endometrium by a proteomics-based analysis. Biochem Biophys Res Commun 2015, 461:211-216

35. Yoshie M, Kashima H, Bessho T, Takeichi M, Isaka K, Tamura K: Expression of stathmin, a microtubule regulatory protein, is associated with the migration and differentiation of cultured early trophoblasts. Hum Reprod 2008, 23:2766-2774

36. Buckley CD, Tan J, Anderson KL, Hanein D, Volkmann N, Weis WI, Nelson WJ, Dunn AR: Cell adhesion. The minimal cadherin-catenin complex binds to actin filaments under force. Science 2014, 346: 1254211

37. Agostinis C, Bulla R, Tripodo C, Gismondi A, Stabile H, Bossi F, Guarnotta C, Garlanda C, De Seta F, Spessotto P, Santoni A, Ghebrehiwet B, Girardi G, Tedesco F: An alternative role of C1q in cell migration and tissue remodeling: contribution to trophoblast invasion and placental development. J Immunol 2010, 185:4420-4429

38. Cohen M, Meisser A, Haenggeli L, Bischof P: Involvement of MAPK pathway in TNF-alpha-induced MMP-9 expression in human trophoblastic cells. Mol Hum Reprod 2006, 12:225-232

39. Hu YX, Dutz JP, MacCalman CD, Yong P, Tan RS, von Dadelszen P: Decidual NK cells alter in vitro first trimester extravillous cytotrophoblast migration: a role for IFN-gamma. J Immunol 2006, 177: $8522-8530$

40. Mor G, Abrahams VM: Potential role of macrophages as immunoregulators of pregnancy. Reprod Biol Endocrinol 2003, 1:119

41. Satoh T, Kidoya H, Naito $H$, Yamamoto $M$, Takemura $N$, Nakagawa K, Yoshioka Y, Morii E, Takakura N, Takeuchi O, Akira S: Critical role of Trib1 in differentiation of tissue-resident M2-like macrophages. Nature 2013, 495:524-528

42. Sica A, Mantovani A: Macrophage plasticity and polarization: in vivo veritas. J Clin Invest 2012, 122:787-795

43. Zhang $\mathrm{W}, \mathrm{Xu} \mathrm{W}$, Xiong S: Macrophage differentiation and polarization via phosphatidylinositol 3-kinase/Akt-ERK signaling pathway conferred by serum amyloid P component. J Immunol 2011, 187:1764-1777

44. Jones RL, Stoikos C, Findlay JK, Salamonsen LA: TGF-beta superfamily expression and actions in the endometrium and placenta. Reproduction 2006, 132:217-232

45. Lim KJ, Odukoya OA, Li TC, Cooke ID: Cytokines and immunoendocrine factors in recurrent miscarriage. Hum Reprod Update 1996, 2:469-481

46. Renaud SJ, Macdonald-Goodfellow SK, Graham CH: Coordinated regulation of human trophoblast invasiveness by macrophages and interleukin 10. Biol Reprod 2007, 76:448-454 